\title{
Horizontal transmission of the ectoparasite Gyrodactylus arcuatus (Monogenea: Gyrodactylidae) to the next generation of the three-spined stickleback Gasterosteus aculeatus
}

\author{
Jaakko Lumme ${ }^{1}$ and Marek S. Ziętara ${ }^{2}$ \\ ${ }^{1}$ Department of Ecology and Genetics, University of Oulu, Finland; \\ ${ }^{2}$ Faculty of Biology, University of Gdańsk, Poland
}

\begin{abstract}
In the parthenogenetic monogeneans of the genus Gyrodactylus Nordmann, 1832, the genetic diversity within or between hosts is determined by the relative roles of lateral transmission and clonal propagation. Clonality and limited transmission lead to high-amplitude metapopulation dynamics and strong genetic drift. In Baltic populations of the three-spined stickleback Gasterosteus aculeatus Linnaeus, the local mitochondrial diversity of Gyrodactylus arcuatus Bychowsky, 1933 is very high, and spatial differentiation weak. To understand the transmission dynamics in a single location, the transmission of the parasite from adults to next generation sticklebacks was investigated in a northern Baltic brackish water location. By sequencing $777 \mathrm{nt}$ of coxl, as many as 38 separate mitochondrial haplotypes were identified. In August, the intensity of gyrodactylid infection on adult hosts was high, the haplotype diversity (h) was extreme and differentiation between fish was negligible (total $h=0.926$, mean $h=0.938$ ). In October, only $46 \%$ of the juvenile sticklebacks carried G. arcuatus. The number of parasites per young fish followed a Poisson distribution $0.92 \pm 1.04$ (mean \pm SD) on October 2, and was clearly overdispersed $2.38 \pm 5.00$ on October 25 . The total haplotype diversity of parasites on juveniles was nearly as high as in adults $(h=0.916)$, but the mean per fish was only $h=0.364\left(F_{S T}=0.60\right)$, due to low intensity of infection and rapid clonal propagation of early arrivals. The initial first come first served advantage of the first gyrodactylid colonisers will be lost during the host adulthood via continuous transmission. Nesting and polygamy are suggested as factors maintaining the high genetic diversity of the parasite population. The transmission dynamics and, consequently, the population structure of Baltic G. arcuatus is fundamentally different from that of G. salaris Malmberg, 1957, on the Baltic salmon Salmo salar Linnaeus.
\end{abstract}

Keywords: clonal propagation, competition, parasite transition

This article contains supporting information (S1-S4) online at http://folia.paru.cas.cz/suppl/2018-65-006.pdf

Some important aspects of the population genetics and evolution of parasites are fundamentally different from those of free-living organisms. The metapopulation dynamics among demes on single hosts in many parasite groups can be extreme, due to the complicated sequence of host generations and randomness in the transition process. The hosts represent ephemeral resource units. If the parasite is not vertically transmitted, the new host generation is born uninfected, and the parasites have - actively or passively - to locate and infect new host individuals. The death of a host means the end of the resource patch. The transition between host generations is critical, and the life cycle of the parasite should be adapted to this transition.

From the point of view of the parasite, some hosts represent coarse-grained and risky resources, others are finegrained and/or predictable (MacArthur and Levins 1964). The challenge of finding a suitable new host is most of- ten overcome by having a very large number of randomly spread propagules. The main characteristics of the host which determine the metapopulation structure and the strategy of the parasite are: (i) the carrying capacity of a single host specimen, i.e. the patch size; (ii) the longevity of the host, relative to the turnover rate of the parasite (patch duration); (iii) the spatial and temporal distance of the host individuals; and (iv) the host immunity. Parasitic factors which determine metapopulation structure and parasite strategy include the dispersal capacity and length of the dispersal phase of the parasite. The parasites often have a phase of asexual multiplication between sexual stages.

The simple and direct transmission biology of hyperviviparous, parthenogenetic and hermaphroditic monogenean flatworms of the genus Gyrodactylus Nordmann, 1832 was well outlined by Boeger et al. (2005), as well as in Chapter 3.3 in Bakke et al. (2007). Gyrodactylus para-

Address for correspondence: M.S. Ziętara, Department of Molecular Evolution, Faculty of Biology, University of Gdańsk, ul. Wita Stwosza 59, 80-308 Gdańsk, Poland. Phone: +48 5852360 50; Fax: +48 5852360 02; E-mail: marek.zietara@biol.ug.edu.pl 
sites are intermediate between micro- and macroparasites (Bakke et al. 2002, 2007). The progeny of these ectoparasites are born as full-grown individuals, one at a time (Cable and Harris 2002). Their hyperviviparity sets strict limits to the dispersal capacity and defines the life cycle. The straight single-host, adult-only life cycle contains no distinct phases for dispersal or dormancy but, at any time during their life, the worms are able to switch from one host individual to another (Boeger et al. 2005).

One of the remaining questions is the relative importance of transmission between host individuals and/or generations, and the clonal propagation of gyrodactylids. In the Gyrodactylus model, the transmission is direct, but subsequent parthenogenetic clonal propagation may lead to amplified metapopulation dynamics, high clonal rarefaction and consequential loss of genetic variation. For example, in a study of Gyrodactylus salaris Malmberg, 1957, a parasite of Atlantic salmon Salmo salar Linnaeus, the populations were strongly structured, apparently by genetic drift (Kuusela et al. 2009). In each of the isolated salmon spawning rivers in the great lake basins of Onega and Ladoga in Russian Karelia, only one parasite mitochondrial haplotype was observed. There is no transmission between adults feeding in the open lakes. The parasite populations are maintained by various juvenile cohorts that inhabit different rivers.

In the large Baltic Tornio River, the population of the parasite G. salaris carried five mitochondrial haplotypes, distributed unevenly in the upper and lower parts of the $460 \mathrm{~km}$ transect during ten years of investigation. The spatial differentiation was $F_{S T}=0.534$ (Lumme et al. 2016a). G. salaris infected $33 \%$ of the sedentary juveniles (parr) and $82 \%$ of the sea-migrating juveniles (smolts). The local parr populations were apparently not infected via the migrating adults or smolts, but the infection was maintained in the nursery rapids via transmission between sedentary juveniles aged 0-4 years (Lumme et al. 2016a). The older parr were more often infected than the first year fry (Anttila et al. 2008). The smolts arriving at the river mouth still carried parasite clones from home, and parasite exchange was limited even in the crowded smolt trap (Lumme et al. 2016a). The conclusion is that spatial genetic differentiation is maintained by local coadaptation of the host and parasite despite the migration of fish from the upper to the lower Tornio River.

In parasites species infecting marine fish, the opportunity for genetic migration between populations allows these parasites to reach populations which are an order of magnitude larger than those on freshwater fish. Consequently, the genetic variation is expected to be higher. In coastal samples around the North Sea, the cox2 of Gyrodactylus gondae Huyse, Malmberg et Volckaert, 2004, on the goby host Pomatoschistus minutus (Pallas), showed haplotype diversity $(h)$ between 0.624 to 0.784 , and the total number of detected haplotypes was 30 (Huyse et al. 2017). This is twice the number of known cox 1 haplotypes in all known salmon-specific populations of $G$. salaris (see Lumme et al. 2016a).
The Varjakka population of Gyrodactylus arcuatus Bychowsky, 1933 analysed here carried as many as 38 cox 1 haplotypes, and the differentiation between Varjakka and Närpiö populations situated $270 \mathrm{~km}$ apart was negligible $\left(\Phi_{S T}=0.011\right.$, Lumme et al. 2016b). The differences observed between $G$. salaris and $G$. arcuatus or $G$. gondae may be explained by differing connectivities of the parasite populations caused by their differing capacities for host-dependent migration.

Here, we introduce and test an amenable system to study the transmission of $G$. arcuatus to the next generation of host in the wild. In the brackish water coastal habitats of the Baltic Sea, the three-spined stickleback (Gasterosteus aculeatus Linnaeus) forms dense and continuous populations (Cano et al. 2008, DeFaveri et al. 2013). Its specific parasite G. arcuatus is also common and numerous, as well as being genetically diverse (Ziętara et al. 2008, Lumme et al. 2016b). Due to the high local mitochondrial diversity of the parasite, transmission can be studied at the level of haplotypes. We will show that the transmission between host generations may present a model for studying the population dynamics of the parasite, which is important for understanding the evolution and coevolution of the host-parasite system. The three-spined stickleback is a superb model for studying evolution (e.g. Schluter 2000, Barber 2013). Studies of the parasites, which have a shorter generation time, might also add an interesting dimension to the understanding of the biology of the host (Criscione et al. 2005; Huyse et al. 2005; Lumme et al. 2016b).

\section{MATERIALS AND METHODS}

The parasite material investigated here was partly utilised for other purposes earlier. The global population structure and phylogeography of Gyrodactylus arcuatus was previously reported (Lumme et al. 2016b). In that paper, the Varjakka material was presented as just one population, without specifying individual hosts. The details of the genetic structure among old and young host individuals, and the transmission from adults to the next generation of sticklebacks is reported here. Knowing the details also provides an important lesson for parasite sampling: fewer adults are needed than juveniles for approaching a correct picture of the local genetic diversity.

\section{Sampling of parasites on old and young fish}

Seven adult sticklebacks (fish cohort OLD, length 57-61 mm, $\mathrm{N}=34$ ) were sampled at the end of the breeding season on 26 August 2004 in Varjakka, Bothnian Bay coast (64.9213N; 25.1019E), Finland. At this time of the year the remaining adults were postreproductive, under a heavy parasite load, often swimming on the surface and expected to be imminently consumed by the next avian host of the cestode Schistocephalus Creplin, 1829. The parasite load included thousands of Gyrodactylus covering most of the surface of the fish (Fig. 1), in addition to the cestode Schistocephalus solidus (Müller, 1776) in the abdomen, the bivalve glochidia in the skin, and the crustacean Argulus foliaceus Linnaeus, 1758 and many small leeches (Hirudinae) on the skin. No quantitative characterisation of the total parasite load was attempted. A sample of G. arcuatus $(\mathrm{N}=20)$ from four adult fish 


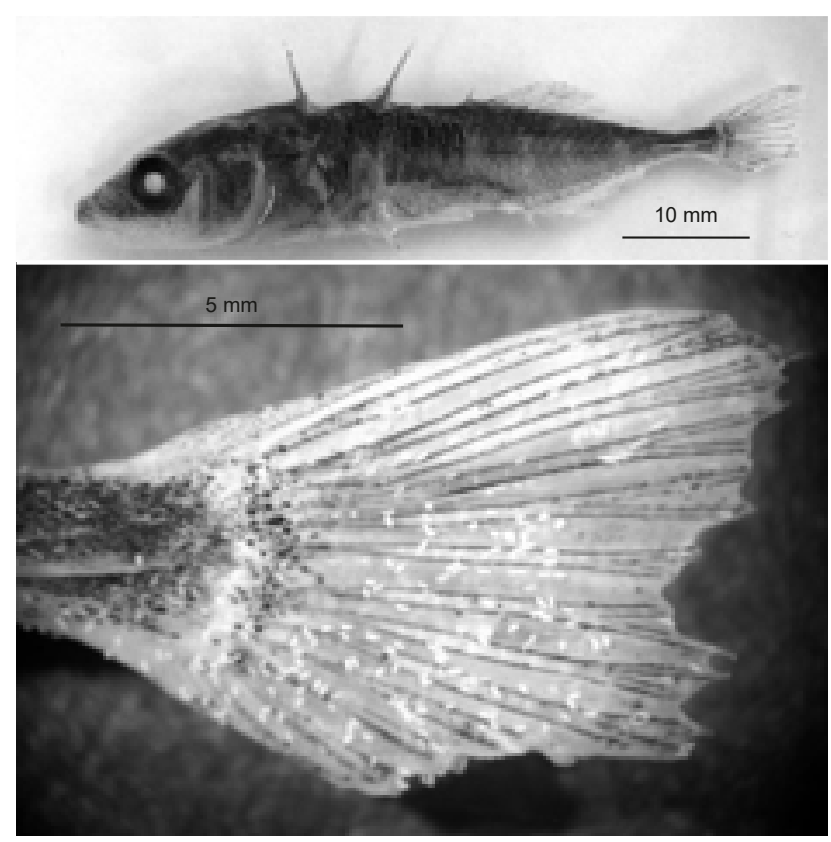

Fig. 1. Postreproductive female Gasterosteus aculeatus Linnaeus No. 2 and its caudal fin, showing the large number of Gyrodactylus arcuatus Bychowsky, 1933 waiting to be transmitted to the next generation.

in Närpiö, $270 \mathrm{~km}$ south along the coast was compared with the Varjakka material.

The first year juvenile fish (fish cohort YOUNG, length $18-27 \mathrm{~mm}, \mathrm{~N}=69$ ) were collected in the same spot as the adults in Varjakka. All fish were killed at site and stored in 96\% ethanol. Thirteen fish were caught in 2 October 2004 (fish length $18-23 \mathrm{~mm}$ ), with a parasite prevalence of $54 \%$. The infected fish carried only one to three parasites each. The second juvenile fish sample was taken three weeks later, on 25 October 2004. It contained 24 juveniles, of length $19-27 \mathrm{~mm}$. In the second sample the parasite prevalence was $42 \%$.

In the adults, only a small sample (5) of the parasites was sequenced from each fish (35 altogether), but in the juveniles, every single parasite specimen (73 altogether) was identified by the $777 \mathrm{nt}$ mitochondrial haplotype (Lumme et al. 2016b).

We adopted a convention to name the parasite individuals as 'haplotypes', to avoid more complicated expressions, e.g. seven 'A28 haplotypes', abbreviating the expression seven 'individuals of haplotype A28'.

\section{DNA utilisation}

The barcoding of the $777 \mathrm{nt}$ fragment of mitochondrial DNA was conducted and the global mitochondrial variation was reported earlier (Lumme et al. 2016b). The phylogeny of the haplotypes in Varjakka is presented in Supplementary file S1. The Fasta file of the Varjakka haplotypes is in Supplementary file S2. GenBank accession numbers of utilised sequences: G. arcuatus (DQ078701DQ078719 and EU862681-EU862704), G. lucii Kulakovskaya, 1952 (EF446749-EF446757 and EU304817-EU304824), G. salaris (AF479750, AF540891, AF540892, AF540899, AF540901, AF540903, AF540905, AF540906, EF117889, EF495063, EF570120, EF612464, AY472084, AY472085, AY840222, DQ180333, DQ468128, DQ988931, DQ993189, DQ993191,
DQ993193-DQ993195， EU223246， EU304825，KT344124KT344126).

Gyrodactylus turnbulli Harris, 1986 from an aquarium guppy Poecilia reticulata Peters in Poland was used as the outgroup. DNA was isolated by digesting a single parasite specimens in $10 \mu$ of lysis buffer ( $1 \times$ PCR buffer, $0.45 \%$ (v/v) Tween $20,0.45 \%$ (v/v) NP 40 and $60 \mu \mathrm{g} / \mathrm{ml}$ proteinase $\mathrm{K}$ ). The tubes were incubated at $65^{\circ} \mathrm{C}$ for $25 \mathrm{~min}$ to allow proteinase $\mathrm{K}$ digestion, then at $95^{\circ} \mathrm{C}$ for $10 \mathrm{~min}$ to denature the proteinase, and then cooled to $4^{\circ} \mathrm{C}$. Aliquots of $2 \mu \mathrm{l}$ of this lysate were used as templates for PCR amplification. The $20 \mu$ PCR reaction mix consisted of $1 \times$ PCR buffer, $0.2 \mathrm{mM}$ dNTPs, $2 \mathrm{mM} \mathrm{MgCl}, 1 \mu \mathrm{M}$ of each primer, $0.5 \mathrm{U}$ of Taq DNA polymerase (Fermentas, Vilnius, Lithuania) and $2 \mu$ of digested parasite. The cycling profile was $94^{\circ} \mathrm{C}$ for 3 min, 38 cycles of $94^{\circ} \mathrm{C}$ for $40 \mathrm{~s}, 50^{\circ} \mathrm{C}$ for $30 \mathrm{~s}$ and $72^{\circ} \mathrm{C}$ for 1.5 $\mathrm{min}$, and a final extension at $72{ }^{\circ} \mathrm{C}$ for $7 \mathrm{~min}$.

The standard ITS rDNA marker for the molecular species identification was amplified with ITS1 (5'-TTTCCGTAGGTG AACCT-3') and ITS2R (5'-GGTAATCACGCTTGAATC-3') and it was sequenced with ITS1R (5'-ATTTGCGTTCGAGAGACCG-3') and ITS2F (5'-TGGTGGATCACTCGGCTCA-3') primers for molecular species identification (Ziętara et al. 2008). The sequence was deposited in GenBank (EF445942).

Mitochondrial DNA ( 1,600 nt fragment of $\operatorname{cox} 1)$ was amplified with FCox6 (5'-TTGGATCATAAGCGCATYGGTAT-3') and 16SR (5'-CATTTAATCATGATGCAAAAGG-3') primers (Ziętara et al. 2010). Three additional internal primers LA1 (5'-TAATAGGGGGGTTTGGTAA-3'), FCox7 (5'-TTTTCAATAGGTATGGACGT-3') and RCox2 (5'-TAGAGAACATGGCGAACACC-3') were used for sequencing. The sequence was deposited in GenBank (EF446767).

The sequence alignment was made by ClustalW as implemented in the MEGA4 package (Tamura et al. 2004, 2007) and then adjusted to the final length of $777 \mathrm{nt}$. The phylogenetic tree was constructed by Neighbor Joining method based on Maximum Composite Likelihood distances $\left(d_{\mathrm{MCL}}\right)$, correcting upwards the large distances reaching saturation. The bootstrap test was conducted with 1,000 repeats. All irrelevant clades were collapsed for simplification.

\section{Data analysis}

The randomness of the numeric parasite distributions on the juvenile fish were compared with a Poisson distribution $\left(\mathrm{x}=\right.$ mean, $\mathrm{s}^{2}=$ variance $\left.=\mathrm{SD}\right)$ and tested by an F-test: $F=\mathrm{s}^{2} / \mathrm{x}$, $\mathrm{df}_{1}=\mathrm{n}-1, \mathrm{df}_{2}=\infty$. The unbiased haplotype diversity estimate was calculated as $\mathrm{h}=[\mathrm{N} /(\mathrm{N}-1)]\left(1-\Sigma \mathrm{p}^{2}\right)$. The fixation index $F_{S T}$ based on haplotype frequencies was calculated as $F_{S T}=\left(h_{T}-h_{S}\right) / h_{T}$, where $h_{T}$ is the total diversity and $h_{S}$ is the mean diversity in separate samples. The $\Phi_{S T}$, which considers the molecular differentiation, was estimated by ARLEQUIN3.1. The basic diversity indices haplotype diversity $(h)$, nucleotide diversity $(\pi)$ and tests of neutrality (Tajima's $D$ and Fu's $F s$ ) were also calculated using ArLeQuin3.1 (Excoffier et al. 2005).

To estimate the expected number and variance of parasite haplotypes drawn randomly from the base population (null model), a simulation program was written in JustBasic (Supplementary file S3) and run with one thousand repeats. As a base population, we used the overall mtDNA haplotype frequencies in Varjakka OLD and YOUNG pooled. The null hypothesis was that the new 
Table 1. Haplotypes found in Gyrodactylus arcuatus Bychowsky, 1933 in Varjakka population.

\begin{tabular}{lcc}
\hline Haplotypes & OLD & YOUNG \\
\hline A1 & 1 & - \\
A2 & 6 & 7 \\
A4 & 1 & 3 \\
A7 & 1 & - \\
A8 & 1 & - \\
A9 & 7 & 8 \\
A10 & - & 2 \\
A11 & 1 & - \\
A12 & 1 & - \\
A14 & 1 & - \\
A15 & - & 1 \\
A16 & 1 & - \\
A17 & 1 & - \\
A18 & 4 & 14 \\
A19 & 1 & 1 \\
A23 & - & 3 \\
A24 & - & 2 \\
A25 & - & 4 \\
A27 & - & 1 \\
A29 & - & 9 \\
A30 & - & 1 \\
A31 & - & 1 \\
A32 & - & 1 \\
A35 & - & 1 \\
A38 & - & 1 \\
A39 & - & 1 \\
A40 & - & 1 \\
A46 & - & 1 \\
B3 & 1 & - \\
C1 & - & 2 \\
C2 & - & 1 \\
C3 & 2 & - \\
C4 & - & 2 \\
C5 & 1 & - \\
C6 & 1 & - \\
C7 & 1 & - \\
C8 & - & 1 \\
C10 & 1 & - \\
\hline Total 38 & 34 & 69 \\
\hline & - & \\
\hline
\end{tabular}

GenBank accession numbers: DQ078701-DQ078719, EU862681EU862694, EU862696 and EU862699-EU862702. Name of the haplotype (clades A, B and C separated) as in Lumme et al (2016b). Due to high haplotype diversity, only five haplotypes were common on both old and young cohorts, but AMOVA between old and young cohorts shows only $\Phi S T=0.031$, i.e. $3 \%$ molecular differentiation between the cohorts.

colonies are a random sample of the base population, without clonal amplification and consequential bias.

\section{RESULTS}

The mitochondrial variation of the population of Gyrodactylus arcuatus in Varjakka, consisting of 38 haplotypes was very large (Table 1). All detected haplotypes belong to the main clade Euro and to the subclades Baltic A, B and C (Lumme et al. 2016b). The number of each haplotype in the fish cohorts OLD and YOUNG is indicated. Only one specimen of subclade B was observed; this subclade is common in the Southern Baltic (Lumme et al. 2016b).

The local genetic diversity of $G$. arcuatus from Varjakka was compared to the global genetic diversity of $G$. lucii and $G$. salaris (Fig. 2). The local degree of variation in G. arcuatus is about the same as global diversity of $G$. sal- aris (see the height of the triangles) but different from that of $G$. lucii.

In Table 2, the mtDNA haplotypes on each fish are presented. Only a small sample was collected from the OLD cohort: these fish were almost fully covered by G. arcuatus (Fig. 1), and the sample was intended to be a population sample for global phylogeographic analysis.

The parasite sample separately counted from the seven adult fish consisted of 25 singleton haplotypes, three times two identical haplotypes on a single fish and once three identical haplotypes on a single fish. The pooled sample OLD consisted of 19 haplotypes, 15 of which were singletons (present as one individual). The other four haplotypes were represented by 2, 4, 6 and 7 specimens as summed over the seven fish (Table 2). A comparable sample $(\mathrm{N}=20$; Lumme et al. 2016b) from four fish in Närpiö, $270 \mathrm{~km}$ south along the coast, contained 18 singletons and only one haplotype pair. The most numerous haplotypes A2 (on ten fish), A9 (on five fish) and A18 (on seven fish) were found in both populations Varjakka and Närpiö, which were not genetically differentiated by analysis of the molecular variance $\left(\Phi_{S T}=0.011\right.$; Lumme et al. 2016b).

An unbiased haplotype diversity estimate, compensating for the different sample sizes $h=\left(\mathrm{N} /(\mathrm{N}-1)\left(1-\Sigma \mathrm{p}^{2}\right)\right.$, was $h=0.926$ for the cohort OLD and $h=0.916$ for the pooled sample YOUNG. The AMOVA between OLD and YOUNG groups showed small but significant differentiation $\Phi_{S T}=0.03075(\mathrm{P}=0.02703 \pm 0.0194,110$ permutations). As a consequence of the high haplotype diversity, only five haplotypes were observed to be common in the two samples. We still assume that the juveniles get the infection from the common pool of parasites, but this occurs on a random basis. This is a good example of sampling bias in highly variable populations.

In the first sample of juveniles (2 October 2004), seven fish out of 13 were infected. One parasite was doubled (haplotype A4 on fish No. 12). The other three pairs were of different haplotypes, and on fish No. 17 all three parasites were different haplotypes. Obtaining two similar haplotypes on one fish by sampling randomly from a common pool with haplotype diversity $H=0.926$ is rare: $\mathrm{P}=(1-0.926)^{2}=0.0055$. Two specimens of $\mathrm{A} 4$ (frequency $1 / 34$ in the OLD pool) is even less probable: $\mathrm{P}=(1 / 34)^{2}=0.000865$. Thus, it seems that clonal propagation may begin immediately after the transfer.

Three weeks later (25 October 2004) the parasite prevalence was still low (42\%), but the haplotype clones on each infected fish were clearly larger, including 12 specimens of A18 on fish No. 24, nine specimens of A29 on fish No. 36 and seven specimens of A9 on fish No. 42. Other clusters contained 4, 3 and 2 clone mates. Pairs were seen in five cases. Clonal propagation was evident in the latter sample of juvenile fish.

We calculated the prediction of the null hypothesis assuming that the specimens had arrived randomly and independently from the basal population (Fig. 3, Supplementary file S4). The accumulating number of haplotype clones on old fish (open dots) follows the null hypothesis. In the young fish, when $\mathrm{N}$ was increasing, there were fewer hap- 


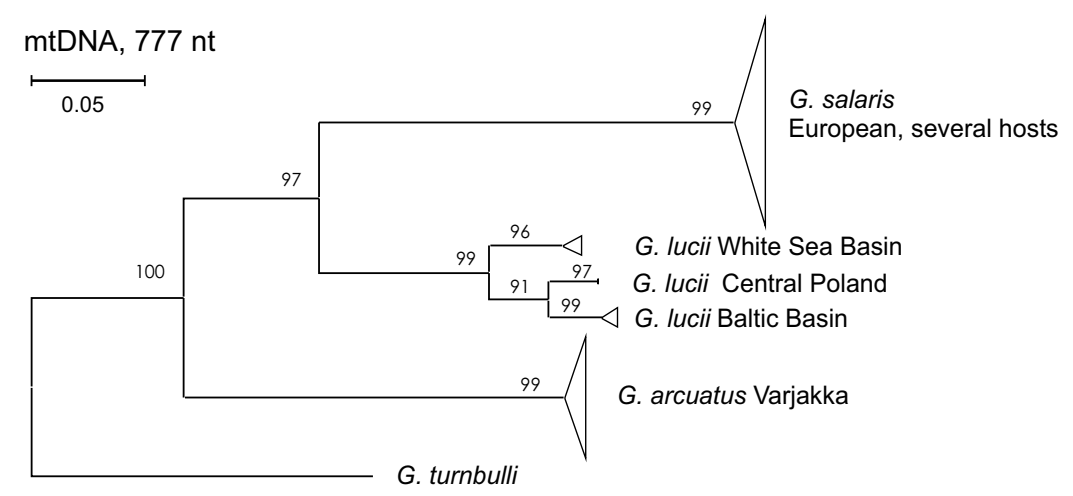

Fig. 2. Comparison of Gyrodactylus arcuatus Bychowsky, 1933 haplotype diversity in the local population of Varjakka with global haplotype diversity of Gyrodactylus lucii Kulakovskaya, 1952 and Gyrodactylus salaris Malmberg, 1957. The outgroup is Gyrodactylus turnbulli Harris, 1986 from guppy Poecilia reticulata Peters aquarium, Poland. The height of the triangles (collapsed clades) indicates the genetic variation. Numbers by nodes indicate bootstrap supports for 1000 repeats.

Table 2. Clonal diversity of Gyrodactylus arcuatus Bychowsky, 1933 on one cohort of adult (OLD) and on two cohorts of juvenile (YOUNG) sticklebacks in Varjakka, the Bothnian Bay.

\begin{tabular}{|c|c|c|c|c|c|c|}
\hline & Fish & Haplotype & $\mathrm{N}$ & $\mathrm{N}_{\mathrm{h}}$ & $\mathrm{h}$ & mean $\mathrm{h}$ \\
\hline \multirow{7}{*}{$\begin{array}{l}\text { OLD } \\
26.08 .2004\end{array}$} & No. 1 & $\mathrm{~A} 1, \mathrm{~A} 2, \mathrm{~A} 18$ & 3 & 3 & 1.000 & \\
\hline & No. 2 & $\mathrm{~A} 2, \mathrm{~A} 4, \mathrm{~A} 18,2 \times \mathrm{C} 3$ & 5 & 4 & 1.000 & \\
\hline & No. 3 & $2 \times \mathrm{A} 2,2 \times \mathrm{A} 9, \mathrm{C} 5, \mathrm{C} 6$ & 6 & 4 & 0.867 & \\
\hline & No. 4 & $\mathrm{~A} 2, \mathrm{~A} 7, \mathrm{~A} 8, \mathrm{~A} 9, \mathrm{C} 10$ & 5 & 5 & 1.000 & \\
\hline & No. 5 & $3 \times \mathrm{A} 9, \mathrm{~A} 11, \mathrm{~A} 12$ & 5 & 3 & 0.700 & \\
\hline & No. 6 & $\mathrm{~A} 2, \mathrm{~A} 14, \mathrm{~A} 18, \mathrm{~B} 3, \mathrm{C} 7$ & 5 & 5 & 1.000 & \\
\hline & No. 7 & A9, A16, A17, A18, A19 & 5 & 5 & 1.000 & \\
\hline Total OLD & & & 34 & 29 & 0.926 & 0.938 \\
\hline \multirow{7}{*}{$\begin{array}{l}\text { YOUNG } \\
02.10 .2004\end{array}$} & No. 10 & A2 & 1 & 1 & 0 & \\
\hline & No. 12 & $2 \times \mathrm{A} 4$ & 2 & 1 & 0 & \\
\hline & No. 14 & A31 & 1 & 1 & 0 & \\
\hline & No. 15 & A18, A32 & 2 & 2 & 1.000 & \\
\hline & No. 17 & $\mathrm{~A} 10, \mathrm{~A} 27, \mathrm{~A} 35$ & 3 & 3 & 1.000 & \\
\hline & No. 18 & A2, A18 & 2 & 2 & 1.000 & \\
\hline & No. 20 & A4 & 1 & 1 & 0 & \\
\hline \multirow[t]{10}{*}{25.10 .2004} & No. 23 & A15 & 1 & 1 & 0 & \\
\hline & No. 24 & $\mathrm{~A} 2,12 \times \mathrm{A} 18,4 \times \mathrm{A} 25, \mathrm{~A} 38,2 \times \mathrm{C} 4$ & 20 & 5 & 0.616 & \\
\hline & No. 31 & A10 & 1 & 1 & 0 & \\
\hline & No. 36 & $9 \times \mathrm{A} 29$ & 9 & 1 & 0 & \\
\hline & No. 37 & A39 & 1 & 1 & 0 & \\
\hline & No. 39 & A46 & 1 & 1 & 0 & \\
\hline & No. 40 & A9, A40 & 2 & 2 & 1 & \\
\hline & No. 41 & $2 \times \mathrm{A} 2$ & 2 & 1 & 0 & \\
\hline & No. 42 & $2 \times \mathrm{A} 2,7 \times \mathrm{A} 9,3 \times \mathrm{A} 23, \mathrm{C} 2$ & 13 & 4 & 0.679 & \\
\hline & No. 43 & $\mathrm{~A} 19,2 \times \mathrm{A} 24, \mathrm{~A} 30,2 \times \mathrm{C} 1, \mathrm{C} 8$ & 7 & 5 & 0.905 & \\
\hline Total YOUNG & & & 69 & 33 & 0.916 & 0.364 \\
\hline
\end{tabular}

$\mathrm{N}$ - number of parasites studied; $\mathrm{N}_{\mathrm{h}}$ - number of haplotypes; $\mathrm{h}$ - haplotype diversity.

Table 3. Neutrality tests Tajima's $D$ and Fu's $F_{S}$ in parasites on OLD and YOUNG fish cohorts.

\begin{tabular}{llll}
\hline Statistics & & OLD & YOUNG \\
\hline Tajima's $D$ test & Sample size & 34 & 69 \\
& S (variable sites) & 42 & 48 \\
& $\pi$ (mean difference) & 6.080 & 4.278 \\
& Tajima's $D$ & -1.476 & -1.890 \\
& $\mathrm{P}(D \neq 0)$ & 0.065 & 0.008 \\
\hline Fu's $F_{S}$ test & No. of alleles & 18 & 24 \\
& $\pi$ & 6.080 & 4.278 \\
& Exp. no. of alleles & 11.904 & 12.644 \\
& $F_{S}$ & -4.228 & -8.440 \\
& $\mathrm{P}\left(F_{S} \neq 0\right)$ & 0.065 & 0.002 \\
\hline
\end{tabular}

lotypes than expected, so we conclude that the parasites had immediately started propagation on the new host, multiplying the number of their mitochondrial haplotype. Most probably, this increase in numbers is clonal, but the alternative that the propagation is sexual but matrilinear cannot be rejected. On the other hand, the juvenile fish No. 43 carried five haplotypes among seven parasites, very close to the number expected by chance $(\mathrm{P}=0.44)$.

Both neutrality tests of molecular variation gave parallel results (Table 3). Tajima's $D$ was negative and significant in the YOUNG cohort $(D=-1.890, \mathrm{P}=0.008)$. Fu's $F_{\mathrm{S}}$ was also large and significant in this cohort $\left(F_{\mathrm{S}}=-8.44\right.$, $\mathrm{P}=0.002)$. The expected versus observed numbers of hap- 


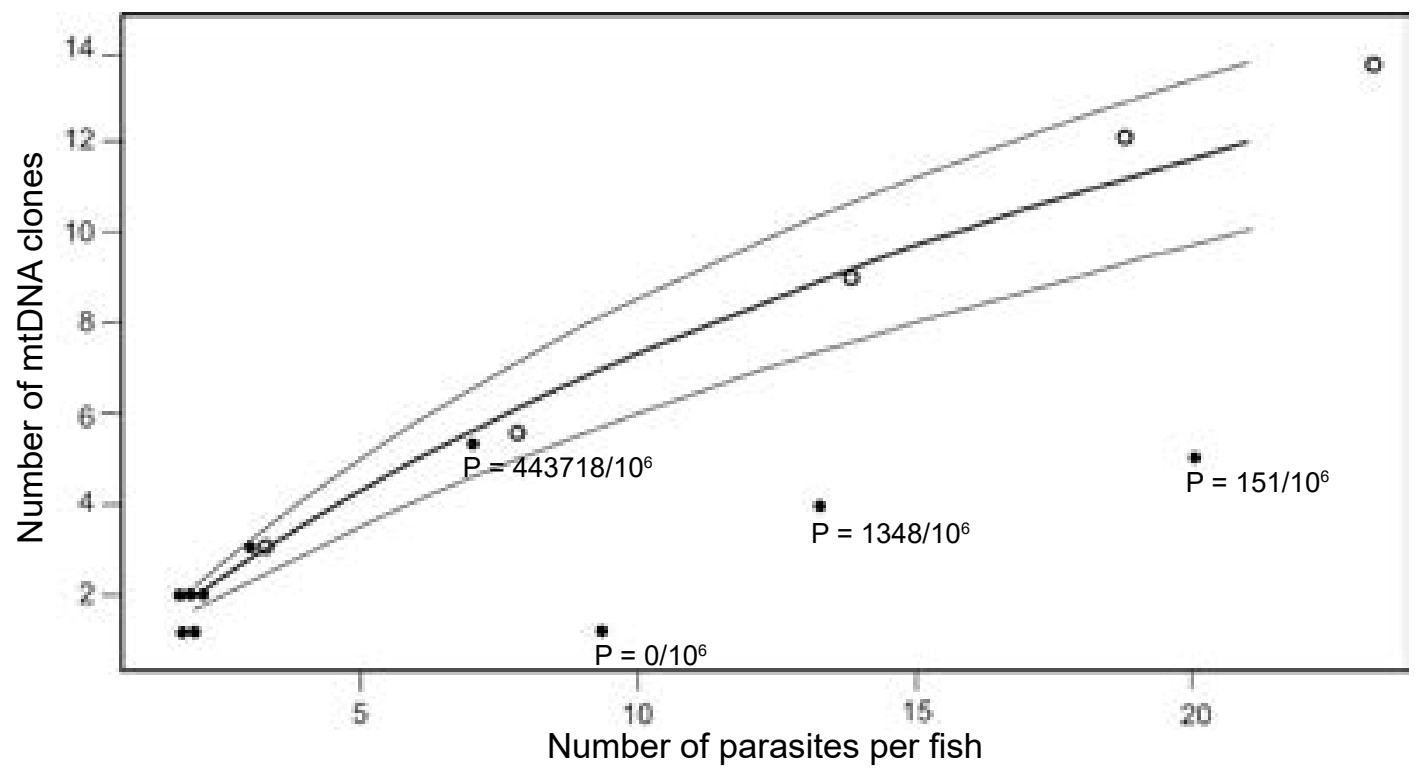

Fig. 3. The mtDNA clone diversity of Gyrodactylus arcuatus Bychowsky, 1933 on the adults (accumulating curve and open dots) and on the individual juveniles of the three-spined sticklebacks studied. The black dots indicate the observed numbers of mtDNA haplotypes as a function of the number of parasites on each juvenile. The curves depict the null hypothesis: expected clone number $($ mean \pm SD) if drawn randomly from the overall frequency distribution of the 38 haplotypes in Varjakka. The $\mathrm{P}$ values on each closed dot indicate the probability of deviation from the null hypothesis. Open dots represent the accumulation of haplotypes when counted from old fish, from specimens Nos. 1-5 (Table 2).

lotypes calculated in Fu's test were illustrative: in the OLD cohort 11.9 haplotypes were expected, but 18 were observed, while in the YOUNG cohort the ratio was higher: 12.6 expected vs 24 observed. As a conclusion, in the total Varjakka population there were many more haplotypes present than predicted by the neutral model based on the molecular indices variable nucleotide sites (S) and nucleotide diversity $(\pi)$. However, on the individual fish of the YOUNG cohort, the tendency was the opposite: fewer haplotypes were present than expected by random sampling from the common pool.

\section{DISCUSSION}

\section{The clonality after colonisation proved?}

It might be of interest to point out that the capacity for clonal propagation of species of Gyrodactylus is usually taken for granted, but seldom explicitly proved. Harris (1998) demonstrated the option and significance of clonality of G. gasterostei Gläser, 1974 by preventing the male maturation for up to 15 generations in aquarium culture. This species belongs to the wageneri group and is not closely related to G. arcuatus. Cable and Harris (2002) considered the clonality as an option to be used in the beginning of infection in a new host, but they also supposed that sexual reproduction is useful in different points of the life cycle of species of Gyrodactylus in general. A lack of suitable markers has so far prevented serious testing of these hypotheses (Bakke et al. 2007).

By using polymorphic nuclear and mitochondrial DNA markers, the importance of clonality was obvious in $\mathrm{G}$. salaris on wild Atlantic salmon populations in the Tornio River area of the Baltic basin (Lumme et al. 2016a). Our genetic marker used in the present study was mitochon- drial DNA, which itself is clonal. Thus, the strict clonality of the propagation of $G$. arcuatus has still to be taken as a plausible hypothesis. A clone of mtDNA (matriline) is automatically produced by sexual means, either by selfing or crossbreeding, but the most appropriate explanation for mitochondrial clones observed in this study on single fish individuals is parthenogenetic propagation. This prediction is mainly made based on three assumptions: (1) asexual reproduction should normally prevail in a hyperviviparous organism as being faster, (2) selfing should be avoided as having serious negative consequences for the offspring, and (3) there is an obvious lack of other sexual partners in an initial colony.

The local vs global genetic diversity is different in G. arcuatus and G. salaris (see Kuusela et al. 2007, Lumme et al. 2016b). The total global degree of variation is about the same in both species (Fig. 2). If the rate of molecular evolution were similar in both species it would suggest that they have accumulated mutations in mitochondrial DNA after a nearly simultaneous bottleneck. However, the geographic and host-specific distribution of this variation is fundamentally different in the two species. In $G$. arcuatus, the coastal Baltic populations are extremely variable and not differentiated, leading to the conclusion that the total parasite population is very large and well-connected (Lumme et al. 2016b). In G. salaris, each local freshwater population is nearly monomorphic, but the separate populations are differentiated. Although the salmon adults share the feeding areas in the open Baltic or in the large lakes, parasite transmission does not happen among them in feeding grounds and therefore they are not transferred between the nursery rapids (Lumme et al. 2015, 2016a). 
The Baltic coastal populations of G. arcuatus are very effectively connected. Cano et al. (2008) also reported weak genetic differentiation among the host, especially among the females, which then may disperse better. Of course, the parasite takes advantage of the most dispersing sex or age group. The dispersal capacity of the parasite has selective interference with virulence, via inclusive fitness: less dispersing phenotypes of the parasite should be less virulent (Wild et al. 2009). In a comparison of G. salaris and $G$. arcuatus, this prediction is broadly followed. Among the resistant Baltic salmon populations, G. salaris is endemic. The parasite does not disperse effectively and the infection is always subclinical of very low parasite intensity (Kuusela et al. 2009, Lumme et al. 2016a). However, the high intensity infection in very old three-spined stickleback adults (Fig. 1) might be a means for the parasite to guarantee the transmission to the next generation, having no longer much influence on the fitness of the fish.

From the point of view of the parasite, the nesting behavior of the three-spined stickleback, which facilitates parasite transmission between adults, might be the key to success. As the male fish attracts sequential females into the nest, and delivers sperm on the eggs immediately, the parasite exchange among the males and females of the whole breeding population must be continuous, and may have equalised the ectoparasite load of all seven mature fish $\left(F_{S T}=0.0\right)$. The parasite colonies on young fish may remain differentiated until sexual maturation. If this model could be proven experimentally, gyrodactylosis caused by G. arcuatus should be considered a veneral (sexually transmitted) disease. From the low prevalence of the parasites in the YOUNG cohort of fish, we can conclude that lucky juveniles can swim out from the nest before being infected. This process deserves detailed empirical investigation.

There are several interesting questions raised on the basis of the present results, as well as from what can be derived from earlier ecological literature on stickleback parasites (Raeymaekers et al. 2008, 2011, Eizaguirre et al. 2009, MacColl and Chapman 2011, de Roij et al. 2011). The host response to the ectoparasite load should be of interest. A high intensity of more than 150 specimens of G. alexanderi Mitzelle et Kritsky, 1967 killed small threespined sticklebacks (Lester and Adams 1974). Is death unavoidable? Can the infected juveniles somehow restrict the growth of a parasite colony, or are the heavily infected specimens just dying out? The results of Eizaguirre et al. (2009) suggest a future study of genetic differences between dying and surviving (or clean and highly infected) fish, e.g. with respect to major histocompatibility complex.

Parasite transmission is the starting point when the host resistance or avoidance could develop (Vogwill et al. 2009). Are the olfactory signals of the host, which lead to orientation and attachment of the parasite, crucial to the evolution of resistance? Becoming unattractive or undetectable would be a good strategy for the host, and the parasite then has to follow suit. Why do stickleback juveniles stay in the same habitat as the parents? Could the benefit of paternal care compensate for the risk of becoming infected? The juveniles (in an aquarium) seem to pick the parasites and pieces of damaged fins of the bigger conspecifics (cleaning), as also observed in some other fish species (Tyler 1963). The survival of free-moving G. alexanderi released from G. aculeatus was lowered due to predation by juvenile sticklebacks, so their tiny teeth are able to kill the worms, but predation might also increase the risk of becoming infected (Lester and Adams 1974). Salmon juveniles in the River Pulonga surely consumed (harmless) G. arcuatus when eating small sticklebacks (Ziętara et al. 2008), but is the nutritional value of Gyrodactylus worms worth the risk for stickleback juveniles?

Acknowledgements. The study was supported by The Finnish Academy (grants 63787 and 134592 to JL).

\section{REFERENCES}

Anttila P., Romakkaniemi A., Kuusela J., Koski P. 2008: Epidemiology of Gyrodactylus salaris (Monogenea) in the River Tornionjoki, a Baltic wild salmon river. J. Fish Dis. 31: 373-382.

Bakke T.A., Cable J., Harris P.D. 2007: The biology of Gyrodactylid Monogeneans: The "Russian-Doll Killers". Adv. Parasitol. 64: 161-376.

BaKke T.A., Harris P.D., Cable J. 2002: Host specificity dynamics: observations on gyrodactylid monogeneans. Int. J. Parasitol. 32: 281-308.

BARBER I. 2013: Sticklebacks as model host in ecological and evolutionary parasitology. Trend. Parasitol. 29: 556-566.

Boeger W.A., Kritsky D.C., Pie M.R., Engers K.B. 2005 Mode of transmission, host switching, and escape from the Red Queen by viviparous gyrodactylids (Monogenoidea). J. Parasitol. 91: 1000-1007.

Cable J., HarRis P.D. 2002: Gyrodactylid developmental biology: historical review, current status and future trends. Int. J. Parasitol. 32: 255-280.

Cano J.M., MäKInen H.S., Merilä J. 2008: Genetic evidence for male-biased dispersal in the three-spined stickleback (Gasterosteus aculeatus). Mol. Ecol. 17: 3234-3242.
Criscione C.D., Poulin R., Blouin M.S. 2005: Molecular ecology of parasites: elucidating ecological and microevolutionary processes. Mol. Ecol. 14: 2247-2257.

DeFaveri J., Jonsson P.R., Merilä J. 2013: Heterogeneous genomic differentiation in marine threespine sticklebacks: adaptation along an environmental gradient. Evolution 67: 25302546.

Eizaguirre C., Yeates S.E., Lenz T.I., Kalbe M., Milinski M. 2009: MHC-based mate choice combines good genes and maintenance of MHC polymorphism. Mol. Ecol. 18: 3316-3329.

Excoffier L., Laval G., Schneider S. 2005: Arlequin ver. 3.0: an integrated software package for population genetics data analysis. Evol. Bioinf. Online 1: 47-50.

HARRIS P.D. 1998: Ecological and genetic evidence for clonal reproduction in Gyrodactylus gasterostei Gläser, 1974. Int. J. Parasitol. 28: 1595-1607.

Huyse T., Oeyen M., Larmuseau M.H.D., Volckaert F.A.M. 2017: Co-phylogeographic study of the flatworm Gyrodactylus gondae and its goby host Pomatoschistus minutus. Parasitol. Int. 66: 119-125.

Huyse T., Poulin R., Théron A. 2005: Speciation in parasites: a population genetics approach. Trend. Parasitol. 21: 469-475. 
Kuusela J., Holopainen R., Meinilä M., Anttila P., Koski P., Ziętara M.S., Veselov A., Primmer C.R., Lumme J. 2009: Clonal structure of salmon parasite Gyrodactylus salaris on a coevolutionary gradient on Fennoscandian salmon (Salmo salar). Ann. Zool. Fenn. 46: 21-33.

Kuusela J., Ziętara M.S., Lumme J. 2007: Hybrid origin of Baltic salmon-specific parasite Gyrodactylus salaris: a model for speciation by host switch for hemiclonal organisms. Mol. Ecol. 16: 5234-5245.

LeSter R.J.G., Adams J.R. 1974: Gyrodactylus alexanderi: reproduction, mortality, and effect on its host Gasterosteus aculeatus. Can. J. Zool. 52: 827-833.

Lumme J., Anttila P., Rintamäki P., Koski P. Romakkaniemi A. 2016a: Genetic gradient of a host-parasite pair persisted ten years against physical mobility: Baltic Salmo salar vs. Gyrodactylus salaris. Inf. Genet. Evol. 45: 33-39.

Lumme J., Mäkinen H., Ermolenko A.V., Gregg J.L., Ziętara M.S. 2016b: Displaced phylogeographic signals from Gyrodactylus arcuatus, a parasite of the three-spined stickleback Gasterosteus aculeatus, suggest freshwater glacial refugia in Europe. Int. J. Parasitol. 46: 545-664.

Lumme J., Ozerov M.Y., Veselov A.E., Primmer C.R. 2015: The formation of landlocked populations of atlantic salmon. In: T. Vladic and E. Petersson (Eds.), Evolutionary Biology of the Atlantic Salmon. CRC Press, Boca Raton Fla, pp. 26-43.

MacArthur R., Levins R. 1964: Competition, habitat selection and character displacement in a patchy environment. Proc. Natl. Acad. USA 51: 1207-1210.

MacColl A.D.C., Chapman S.M. 2011: Parasites can cause seelction against migrants following dispersal between environments. Func. Ecol. 24: 847-856.

Raeymaekers J.A.M., Huyse T., Maelfait H., Hellemans B., Volckaert F.A.M. 2008: Community structure, population structure and topographical specialization of Gyrodactylus (Monogenea) ectoparasites living on sympatric stickleback species. Folia Parasitol. 55: 187-196.

Received 10 November 2016

Accepted 17 January 2018
Raeymaekers J.A.M, Wegner K.M., Huyse T., Volckaert F.A.M. 2011: Infection dynamics of the monogenean parasite Gyrodactylus gasterostei on sympatric and allopatric populations of the three-spined stickleback Gasterosteus aculeatus. Folia Parasitol. 58: 27-34.

de Roij J., Harris P.D., MacColl D.C. 2011: Divergent resistance to a monogenean flatworm among three-spined stickleback populations. Func. Ecol. 25: 217-226.

SCHLUter D. 2000: The Ecology of Adaptive Radiation. Oxford University Press, New York, 296 pp.

Tamura K., Dudley J., Nei M., Kumar S. 2007: MEGA4: Molecular Evolutionary Genetics Analysis (MEGA) Software Version 4.0. Mol. Biol. Evol. 24: 1596-1599.

Tamura K., Nei M., Kumar S. 2004: Prospects for inferring very large phylogenies by using the neighbor-joining method. Proc. Natl. Acad. Sci. USA 101: 11030-11035.

TyLER A.V. 1963: A cleaning symbiosis between the rainwater fish, Lucania parva and the stickleback, Apeltes quadracus. Ches. Sci. 4: 105-106.

Vogwill T., Fenton A., Brockhurst M.A. 2009: The impact of parasite dispersal on antagonistic host-parasite coevolution. J. Evol. Biol. 21: 1252-1258.

Wild G., Gardner A., West S.A. 2009: Adaptation and the evolution of parasite virulence in a connected world. Nature 459: 983-986.

Ziętara M.S., Kuusela J. Veselov A., Lumme J. 2008: Molecular faunistics of accidental infections of Gyrodactylus Nordmann, 1832 (Monogenea) parasitic on salmon Salmo salar L. and brown trout Salmo trutta L. in NW Russia. Syst. Parasitol. 69: 123-135.

ZiĘtara M.S., Rokicka M., Stojanovski S., Lumme J. 2010: Introgression of distant mitochondria into the genome of Gyrodactylus salaris: nuclear and mitochondrial markers are necessary to identify parasite strains. Acta Parasitol. 55: 20-28.

Cite this article as: Lumme J., Ziętara M.S. 2018: Horizontal transmission of the ectoparasite Gyrodactylus arcuatus (Monogenea: Gyrodactylidae) to the next generation of the three-spined stickleback Gasterosteus aculeatus. Folia Parasitol. 65: 006. 